\title{
MATERIAL OPTIMIZATION AND DYNAMIC MATERIALS
}

\author{
K.A. Lurie \\ Worcester Polytechnic Institute \\ 100 Institute Road, Worcester, MA 01609, U.S.A
}

Article history:

Received 18.08.2021, Accepted 29.09.2021

\begin{abstract}
The paper is about the connection between material optimization in dynamics and a novel concept of dynamic materials (DM) defined as inseparable union of a framework and the fluxes of mass, momentum, and energy existing in time dependent material formations. An example of a spatial-temporal material geometry is discussed as illustration of a DM capable of accumulating wave energy.

Finding the optimal material layouts in dynamics demonstrates conceptual difference from a similar procedure in statics. In the first case, the original constituents are distributed in space-time, whereas in the second - in space alone. The habitual understanding of a material as an isolated framework has come from statics, but a transition to dynamics brings in a new component - the fluxes of mass, momentum, and energy. Based on Noether theorem, these fluxes connect the framework with the environment into inseparable entity termed dynamic material (DM). The key role of DM is that they support controls that may purposefully change the material properties in both space and time, which is the main goal of optimization.
\end{abstract}

\section{Dedication}

This paper is dedicated to the memory of Iliya Izraelevich Blekhman - an outstanding scholar of the world caliber, one of the founders and main contributors to modern non-linear mechanics. Through many long years, I have been among those who enjoyed his friendship, but through the last two decades, our scientific interests became mutual, and our intensive professional discussions continued until his passing.

These discussions were captivating because we both had a feeling that we had touch based a universal concept in material science and optimization, that developed into what is known to-day as the concept of dynamic materials (DM), i.e., materials with space-time dependent properties. We were mostly concerned with bringing the relevant mathematics as close as possible to the first prin- ciples. A deeper physical understanding of this connection has led to noticeable simplification of solutions to problems of control and optimal design in material dynamics, specifically dealing with formations that exist in space and time.

The original ideas have been published in 2000 in a brief joint paper [Blekhman and Lurie, 2000], developed in subsequent publications (e.g., [Blekhman, 2007; Lurie, 2009; To, 2009; Shui et al., 2014; Shui et al., 2015; Lurie and Weekes, 2006; Lurie et al., 2009]), and summarized in the monograph [Lurie, 2007] published by Springer in two editions (2007 and 2017). Iliya's passion for new ideas always inspired me through this work, and remains in my memory as indefeasible feature of his unforgettable personality.

\section{Materials in Statics and Dynamics}

Material optimization, after several millennia of its progress as an art, began to transform into science - a part of applied mathematics - only several decades ago. A theoretical basis for this transformation has roots in the calculus of variations, with addition of some specifics put forth due to dissimilar applications. The first stage of this development was related to statics, with its mathematical embodiment being the elliptic equations.

These studies introduced composites as an almost inevitable element of optimal design, that received implementation in modern technology. However, even in their modified constructive version, such formations demonstrate limited use because they are not adjusted to the temporal variations of environment. To be able to adequately handle dynamics, a material medium must itself be time-dependent, i.e., its properties should vary in both space and time. Any substance maintaining such variation has been termed a dynamic material (DM). The main difference between conventional materials and DM is that the first exist in space alone, and the second develop in space-time and cannot exist isolated from their environment. This connection is realized through the exchange of mass, momentum, and energy between the 


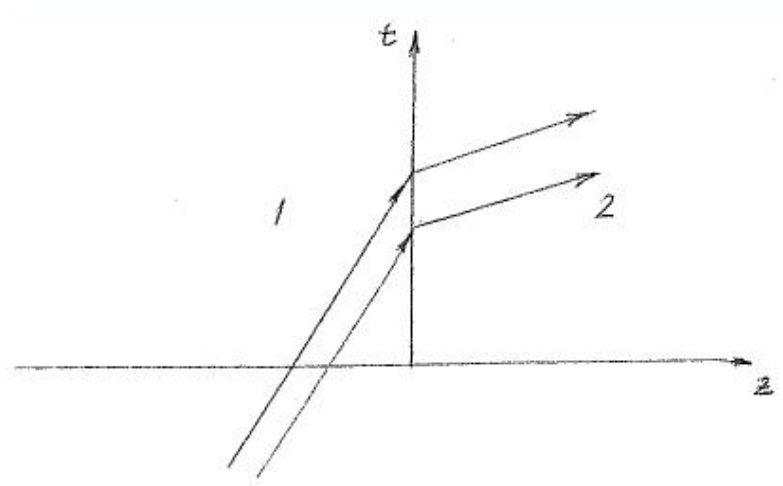

Figure 1.

two media. The well-known Noether Theorem says that for a system with Hamiltonian that depends explicitly on time (be it because of material motion or due to the property change alone, with immovable material framework) - for every such system there is no conservation of energy. This means the existence of energy fluxes between the system and the environment. The fluxes are inseparable from the framework, and their union is precisely what we call a DM. Being a little abstract (this definition goes beyond the stock notion of a material used in everyday language), it nevertheless clearly explains its physical meaning. Particularly, every DM appears to be a thermodynamically open system.

There is an impressive multitude of DM around us, the most remarkable one created by mother Nature as a living tissue. Many men made examples came into being due to recent technologies, e.g., rockets, robots, tunable transmission lines, etc. The energy gain or loss is maintained due to an external agent committing the work that pumps the energy into a system or releases it into the environment. The presence of this agent is an inseparable attribute of any dynamic material.

DM is an entity that exists in space-time, i.e., in pseudo-Euclidean Minkowski space. These formations can be classified into two conceptually distinct categories named activated and kinetic. The activated DM demonstrate the spatial-temporal variation of their material property pattern alone, with no actual motion of a material framework. Contrary to that, the kinetic DM may be heterogeneous in space and involved only in mechanical motion through the property pattern that remains immovable in a laboratory frame.

An interesting illustration of this difference belongs to L.I. Slepyan. He noticed that a non-linear elastic body is always a kinetic DM because its properties are immersed into it as nonlinearities and cannot move independently from the framework. Contrary to that, a linear elastic body may become an activated DM, with its property pattern performing an independent motion.

The spatial-temporal nature of DM means that their properties are transformed by a Lorentz group, contrary to the Euclidean group in statics. Its distinct embodiment is given by electrodynamics of moving bodies where it is appropriate to consider heterogeneous formations assembled from ordinary materials in space-time.

Remark. Consider two points on a temporal interface $t=$ const between two contacting dielectrics in spacetime. None of these points can be interpreted as an event that is a consequence of different event occurred at another point on the same interface because otherwise the causality principle would be violated. This also holds for any two points on any space-like interval for which a temporal boundary is an example. This means that no space-like interval can be a route for a signal, so its slope $d z / d t$ may be arbitrarily large without violation of relativity. This criterion allows for a substantial extension of material geometry in space-time.

\section{Examples of Material Geometries with DM}

Below we discuss several examples of material structures in 1D space plus time, typical for electromagnetic theory but universally applicable elsewhere.

Assume we have two isotropic dielectrics, each with material parameters $\alpha=(\varepsilon \mu)^{-1 / 2}$ and $\gamma=(\mu / \varepsilon)^{1 / 2}$; let their wave impedances $\gamma_{1}$ and $\gamma_{2}$ match $\left(\gamma_{1}=\gamma_{2}\right)$, but the phase velocities $\alpha_{1}$ and $\alpha_{2}$ differ from each other: $\alpha_{1}<\alpha_{2}$, so the second dielectric is fast, and the first is slow. Also, let the dielectrics contact along the time-like plane $z=0$ (the spatial interface). An incident electromagnetic wave travelling through the uniform material 1 along the $z$-axis (i.e., normally to the interface) will then produce no reflected wave moving back into material 1 after it reaches the interface $z=0$. The same holds if we exchange materials 1 and 2 when they contact along the same plane. In both cases, the only secondary wave will be a transmission wave travelling into material 2 in the first case, and into material 1 in the second (Fig. 1).

A similar situation arises if the interface between materials is temporal, say, $t=t_{0}$ (Fig. 2). Once $\gamma_{1}=\gamma_{2}$, the incident wave originally travelling through material 1 generates, as before, only one secondary wave propagating with velocity $\alpha_{2}$ through material 2 in the same direction as the incident wave.

Formally, the wave routes in Figs. 1 and 2 look similar, with no reflections, but they differ from each other in the energy performance. Being continuous at spatial interfaces, the energy changes at temporal interface by factor $\alpha_{2} / \alpha_{1}$; so it increases when $\alpha_{2}>\alpha_{1}$, and decreases otherwise [Morgenthaler, 1958]. Given these observations, we can easily design a material geometry in space-time capable to accumulate energy and concentrate it in progressively narrowing pulses. To achieve that, it is necessary to avoid the energy losses down the road. This means that the wave routes should never enter the slow material from fast across the temporal transients; they may enter it only across the spatial interface where no energy is lost. So all appropriate crossings either leave the energy continuous at spatial interfaces, or add it to 


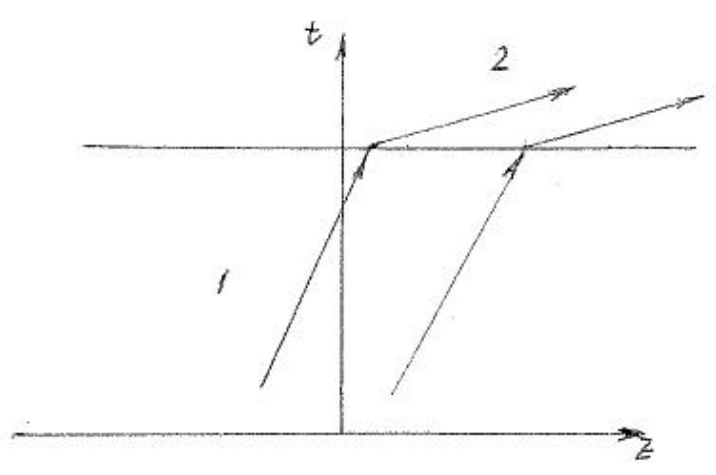

Figure 2.

the wave at temporal transients when crossing them from slow material to fast. This is true for both forward and backward waves that can be discussed independently because of no reflection at both types of transients. So we may discuss, for example, the right going waves.

One should now ask if there exists an assembly of rectangular domains in $(z, t)$-plane that supports the wave routes that ensures the energy accumulation. And here is the key point: a doubly periodic checkerboard property layout (Fig. 3) provides such support for a range of lattice parameters $m_{1}, n_{1}$. A typical property period (see Fig.4) is a square $1 \times 1$, with materials 2 and 1 occupying, respectively, the diagonal rectangles $m_{1} \times n_{1}$, $\left(1-m_{1}\right) \times\left(1-n_{1}\right)$, and $m_{1} \times\left(1-n_{1}\right),\left(1-m_{1}\right) \times n_{1}$. The range for $m_{1}, n_{1}$ has been precisely specified by the sharp bounds determined in [Lurie et al., 2009]. Specifically, for the right going waves in Fig. 3, the energy is accumulated in the array of progressively sharpening pulses (limit cycles) composed from densely compressing wave routes. An attentive reader will find from this figure that the crossings of wave routes with material interfaces become, after a few periods, consistent with the description given above.

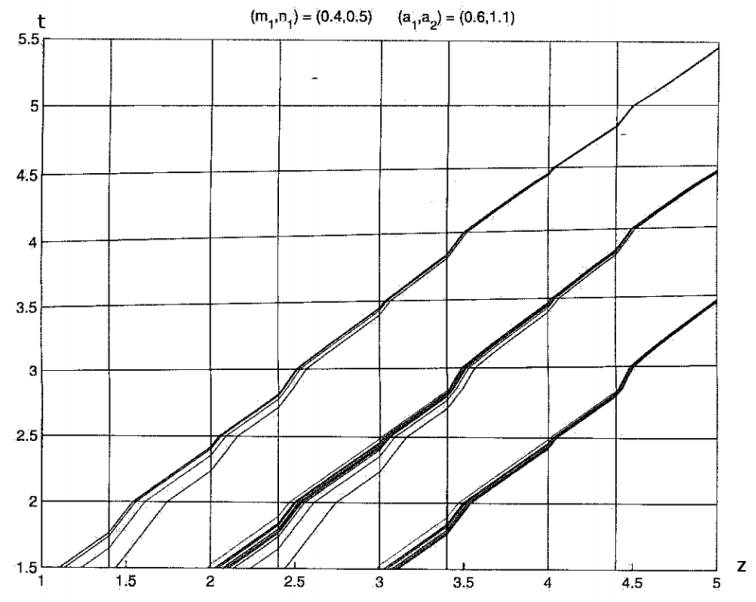

Figure 3. A closer look at limit cycles

This solution is perfectly understandable due its ut- most simplicity that comes from the physical clarity. Its understanding needs no more than the Snell's law plus some attention to the kinematics of the wave routes. The energy gain is hidden in the formulae of Morgenthaler [Morgenthaler, 1958] and its special analysis for our purposes is excessive.

After the original works [Blekhman and Lurie, 2000; Blekhman, 2007; Lurie, 2009; To, 2009; Shui et al., 2014; Shui et al., 2015; Lurie and Weekes, 2006; Lurie et al., 2009; Lurie, 2007] had been published, there began to appear papers of other authors who at last paid their attention to the media with time-dependent properties. Examples of such publications are given by [Bialancalana et al., 2007; Taravati, 2018; Huidobro' et al., 2019]. After long and excessive mathematics, their authors arrived at the results that almost totally duplicate findings published before in [Blekhman and Lurie, 2000; Blekhman, 2007; Lurie, 2009; To, 2009; Lurie and Weekes, 2006; Lurie et al., 2009; Lurie, 2007], but with no reference to these original works as if they had never existed. This fact illustrates one more time how longsighted was Professor Blekhman as he became one of those who first put forth these ideas.

\section{Conclusion}

An idea of dynamic materials is implemented toward finding the material geometry supporting the energy accumulation in a space-time dependent nonhomogeneous formation.

\section{References}

Bialancalana, F., Amann, A., Uskov, A., and O'Reilly, E. P. (2007). Dynamics of light propagation in spatiotemporal dielectric structures. Phys. Rev., E75.

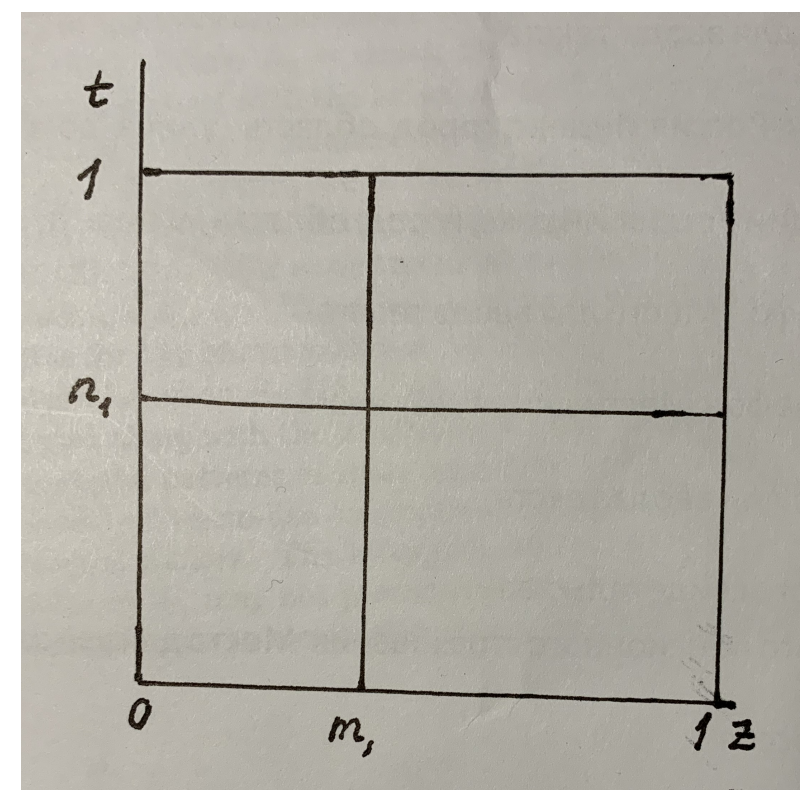

Figure 4. 
Blekhman, I. I. (2007). On vibratory dynamic materials and composites. Doklady Physics, 52 (6), pp. 335-338.

Blekhman, I. I. and Lurie, K. A. (2000). On dynamic materials. Proc. Rus. Acad. Sci. (Doklady), 37, pp. 182185.

Huidobro', P. A., Galiffi, E., Guenneau, S., Craster, R. V., and Pendry, J. B. (2019). Fresnel drag in space - time modulated metamaterials. Proc. Nat. Acad. Sci. USA, 116 (50), pp. 24943-24948.

Lurie, K. A. (2007). An Introduction to the Mathematical Theory of Dynamic Materials. Springer.

Lurie, K. A. (2009). On homogenization of activated laminates in $1 \mathrm{~d}$ - space and time. ZAMM, 89(4), pp. 333-340.

Lurie, K. A., Onofrei, D., and Weekes, S. L. (2009). Mathematical analysis of the wave propagation through a rectangular material structure in space-time. J. Math. Anal. Appl., 355, pp. 180-194.

Lurie, K. A. and Weekes, S. L. (2006). Wave propaga- tion and energy exchange in a spatio-temporal material composite with rectangular microstructure. J. Math. Anal. Appl., 314 (1), pp. 286-310.

Morgenthaler, F. R. (1958). Velocity modulation of electromagnetic waves. IRE Trans Microw Theory Tech., 6, pp. 167-172.

Shui, L.-Q., Yuz, Z.-F., Liu, Y.-S., Liu, Q.-C., and Guo, J.-J. (2014). One-dimensional linear elastic waves at moving property interface. Wave Motion, 51, pp. 1179-1192.

Shui, L.-Q., Yuz, Z.-F., Liu, Y.-S., Liu, Q.-C., Guo, J.-J., and He, X.-D. (2015). Novel composites with asymmetric elastic wave properties. Compos. Sci. Technol., 113, pp. 19-30.

Taravati, S. (2018). Giant linear nonrresiprocity, zero reflection and zero band gap in equilibrated space-time variable media. Phys. Rev. Applied, 9.

To, H. T. (2009). Homogenization of dynamic laminates. J. Math. Anal. Appl., 354 (2), pp. 518-538. 